\title{
Genetic interaction screens advance in reverse
}

\author{
Suzanne Komili ${ }^{1,2,3}$ and Frederick P. Roth ${ }^{1,2,4,5}$ \\ ${ }^{1}$ Department of Biological Chemistry and Molecular Pharmacology, Harvard Medical School, \\ Boston, Massachusetts 02115, USA; ${ }^{2}$ Harvard Biophysics Graduate Program, Harvard Medical School, Boston, Massachusetts \\ 02115, USA; ${ }^{3}$ Department of Systems Biology, Harvard Medical School, Boston, Massachusetts 02115, USA; ${ }^{4}$ Center for \\ Cancer Systems Biology, Dana-Farber Cancer Institute, Boston, Massachusetts 02115, USA
}

A genetic interaction is defined by the emergence of a surprising phenotype when two genes are disrupted together. In 2004, Baetz et al. (2004) reported a new kind of large-scale reverse-genetic screen for genetic interactions. By mating a haploid yeast strain carrying a "query mutation" to many haploid strains-each carrying a single engineered mutation at a second (unlinked) locus-they efficiently produced diploid strains carrying two heterozygous mutations. If the double-mutant strain shows reduced fitness relative to each single-mutant strain, this indicates a genetic interaction traditionally called "unlinked noncomplementation" (UNC). UNC interactions have been noted to frequently occur between genes that encode physically interacting proteins (Stearns and Botstein 1988; Fuller et al. 1989). Although there have been many forward-genetic screens for UNC interactions (Hays et al. 1989; Yook et al. 2001; Hawley and Walker 2003), Baetz et al. (2004) took a reverse-genetic approach-systematically and exhaustively testing a collection of gene pairs for UNC interactions. They screened a heterozygous "query mutation" in CTF13, which encodes a core kinetochore protein, against a library of heterozygous null mutations. They found genetic modifiers of chromosome segregation and also distinguished separable functions within the RSC chromatin remodeling complex.

In this issue of Genes \& Development, Haarer et al. (2007) confirm the value of this new kind of screen and take it one step further. They also coin a new name for the subtype of UNC interaction in which at least one of the mutant loci carries a null mutation, calling it a "complex haploinsufficient" (or CHI) interaction. Haarer et al. (2007) used a null allele of actin as a "query mutation," screening this against virtually all nonessential genes for $\mathrm{CHI}$ interactions. They validate the $\mathrm{CHI}$ interaction screen by identifying $208 \mathrm{CHI}$ interactors, many known to be functionally related to actin. Moreover, they carry the CHI screen one step further, using a panel of query alleles to characterize each "hit" by testing it for interaction with each of six different reduced-func-

${ }^{5}$ Corresponding author.

E-MAIL fritz_roth@hms.harvard.edu; FAX (617) 432-3557.

Article is online at http://www.genesdev.org/cgi/doi/10.1101/gad.1512707. tion (hypomorphic) actin alleles. Different query alleles retrieved different subsets of interactors, indicating that $\mathrm{CHI}$ genetic screens can lead to nuanced information about functional relationships. A large subset of CHIs were with genes that encode ribosomal proteins. Interestingly, the results added to a growing and intriguing body of evidence supporting the idea that many pairs of duplicated ribosomal protein genes perform distinct functions.

\section{Gene dosage and poison models to explain UNC relationships}

At least two models have been put forward to explain the observation of an UNC relationship - the gene dosage model and the poison model (Stearns and Botstein 1988; Fuller et al. 1989). Under the former model, the double mutant's defect is caused by reduced gene dosage at the two alleles, while the latter model requires that at least one of the alleles actively "poisons" some process, sensitizing the organism to a secondary mutation. When both alleles are null mutations, the gene dosage model is the preferred explanation.

The precise level of a gene's expression can have profound effects on its function. This is best illustrated in the case of protein complexes: If expression of a component is too low, complexes lacking that component may be functionally impaired. Conversely, if a component of a given complex is expressed too highly, it can sequester other members into nonfunctional subcomplexes. It is therefore not surprising that components of the ribosome complex are tightly coregulated, both transcriptionally and post-transcriptionally, including complex feedback regulation (Presutti et al. 1991; Vilardell and Warner 1997). By contrast, translation factors lack such sophisticated regulatory mechanisms, and changes in expression levels of translation factors can lead to large increases in translation read-through errors (Le Goff et al. 1997; Salas-Marco and Bedwell 2004).

\section{The trouble with forward genetics}

A central part of forward genetics is the hunt for random mutants with a desired or interesting phenotype. By 
their nature, random mutations have variable effects and unevenly distributed locations, making it difficult to determine when a screen has been performed to saturation. Some tricks can simplify the task of locating the mutant of interest; e.g., screens for dominant or multicopy suppressors can use a library of plasmids with inserted sequences that have been mutagenized or overexpressed, limiting the search space for the causative allele. More recently, tiling arrays have introduced the prospect of rapid and inexpensive resequencing of entire genomes (Gresham et al. 2006). However, even when a mutation has been mapped and sequenced, the probable effect of the mutation on its host gene generally remains uncertain. It is simpler to interpret the phenotype of a null allele, such as a precise gene deletion, than it is to interpret the effects of a missense mutant. Like simpler forward-genetic mutant screens, forward-genetic interaction screens are subject to the challenges of locating and interpreting random mutations.

\section{The awesome power of reverse genetics}

Knowledge of genome sequence coupled with efficient techniques for targeted gene disruption (e.g., homologous recombination in Saccharomyces cerevisiae and RNA interference [RNAi] in other organisms) has enabled the development of tools to make genetic screens much more systematic. $S$. cerevisiae has been an ideal model organism for reverse genetics, especially given a collection of strains that has been developed in which each strain carries a targeted deletion (Winzeler et al. 1999). This strain collection has enabled the systematic identification of genes required for a given cellular function. In some screens, strains are individually assayed for a given defect; for example, in mRNA export (Hieronymus et al. 2004). A more high-throughput approach involves assaying cell growth rates in response to different growth conditions (e.g., drug exposure) to identify genes required for growth (or resistance to a drug) (Begley et al. 2002; Giaever et al. 2004; Dudley et al. 2005; Parsons et al. 2006). Technology allowing rapid readout of competitive growth in pooled cultures of deletion strains has significantly increased the speed and sensitivity of growth rate assays (Giaever et al. 2002).

\section{Reverse-genetic interactions}

Reverse-genetic resources and technologies have also made the analysis of genetic interactions much more tractable on a large scale. These resources, coupled with the development of genetic markers for efficient selection of haploid yeast cells (Tong et al. 2001), have allowed efficient screening for synthetic sick or lethal genetic interactions (defined by a double-mutant with a surprisingly poor growth phenotype). Synthetic genetic array (SGA) analysis has been used to identify global synthetic interaction networks (Tong et al. 2001, 2004). The combination of the deletion strain collection, SGA technology, and bar code microarrays have allowed diploid- based synthetic lethality analysis on microarrays (dSLAM), in which double-mutant strains are efficiently produced and grown competitively in pools. This approach has been used to study more specific networks of genes responsible for DNA integrity (Ooi et al. 2003; Pan et al. 2006). Analogous methods have been used to discover synthetic genetic interactions involving essential genes using the collection of strains with tet-inducible genes (Davierwala et al. 2005) and the DAmP collection with destabilized transcripts (Schuldiner et al. 2005). While synthetic genetic interactions often indicate compensatory or parallel gene action, alleviating interactions-in which the double mutant grows faster than would be expected given single-mutant growth ratesoften indicate serial or concerted gene action. While previous SGA and dSLAM screens have focused on synthetic interactions, more recent screens have captured alleviating interactions (Schuldiner et al. 2005; Collins et al. 2006; R.P. St. Onge, R. Mani, J. Oh, M. Proctor, E. Fung, R. Davis, C. Nislow, F.P. Roth, and G. Giaver, unpubl.).

\section{Reverse-genetic approaches to gene dosage effects}

Reverse genetic approaches can be used to explore the effects of changes in gene dosage. For example, in haploinsufficiency screens, the growth rate among diploids with two functional copies of a gene is compared with diploids carrying only one functional copy. One such screen identified proteins required for optimal growth rate, such as metabolic processes and protein production (Deutschbauer et al. 2005). High-throughput genetic interaction screens have also been carried out to find synthetic dosage lethality interactions, in which the combination of a reduced-function mutation with the overexpression of a second gene causes loss of fitness (Stevenson et al. 2001; Sopko et al. 2006).

The CHI screens by Baetz et al. (2004) and Haarer et al. (2007) can be viewed as a search for haploinsufficiency among null alleles that depends on a "query mutation" at another locus. Several of the CHI interactors, like those found by Deutschbauer et al. (2005), are required for optimal biosynthetic capacity including metabolism, protein synthesis, and transcription. However, there was also clear enrichment for genes that are functionally related to actin, such as those involved in cell organization and biogenesis, as well as many actin-binding proteins. These results illustrate the extent to which CHI interactions allow the elucidation of specific cellular pathways.

The results of CHI screens may have broader implications for multigenic human disease than other types of synthetic genetic interaction screens, because they relate to recessive heterozygotic alleles in a diploid genome. Baetz et al. (2004) found $36 \mathrm{CHI}$ interactions to their query locus (CTF13/ctf13-30), and Haarer et al. (2007) found 208. The proteins encoded by these genes have 13 and 36 protein interactions, respectively, according to BioGRID (Stark et al. 2006), suggesting that the density of $\mathrm{CHI}$ genetic interaction is greater than the 
density of protein interactions. Estimating the precise impact on human disease of UNC and $\mathrm{CHI}$ interactions is difficult. The frequency of disease caused by UNC depends on the probability that two recessive alleles will exhibit an UNC interaction. It also depends on the frequencies of each of the interacting alleles in the human population; indeed, the population frequencies of alleles with UNC interactions may have been driven lower precisely because of the deleterious effect of the UNC interaction, and driven lower still for alleles with many severe UNC interactions such as those observed for actin. However, deleterious recessive alleles can accumulate to appreciable allele frequencies, especially if their effects are "revealed" only in the presence of another recessive allele at a separate locus. Regardless of the frequency of human UNC and CHI interactions, $\mathrm{CHI}$ screens will uncover a new layer of genetic interactions in yeast. This in itself could have an impact on human disease, since many mechanistic insights into biological processes in yeast have shed light on the function of orthologous human disease genes.

Another interesting innovation by Haarer et al. (2007) was the analysis of their genetic interactors with different actin alleles. Although a previous study had identified genetic interactions with a temperature-sensitive allele of TAF9 (Milgrom et al. 2005), Haarer et al. (2007) assayed each of actin's CHI "hits" for genetic interactions with each of six actin alleles. These actin alleles correspond to mutations on the surface of different regions of the actin protein. Each allele exhibited genetic interactions with a subset of actin's CHI interactors, with the more severe alleles exhibiting the most interactions. This assay adds further dimensionality to the space of genetic interactions and thus provides much more information about the $\mathrm{CHI}$ interactors. As the actin alleles used for this analysis are designed to neutralize clusters of charged residues on the surface of the actin protein, probing genetic interactions with these alleles divides the $\mathrm{CHI}$ interactors into groups presumably based on the region at which they interface with actin. This is consistent with Haarer et al.'s finding that the alleles resulting in mutations near each other on the actin protein share a statistically significant number of interactors. It will be exciting to see the levels of biological complexity that further applications of this type of assay will reveal.

\section{Functional divergence among pairs of paralogous ribosomal protein genes}

An ancient ancestor of the yeast $S$. cerevisiae experienced the duplication of its entire genome. Although most of the duplicated genes have since been lost, a subset have remained, playing roles in transcription, intracellular transport, and translation, among others. For most of the retained pairs, one copy has maintained its original function (the "ancestral" copy) and approximate sequence, whereas the other has diverged and now plays a similar, yet specialized, cellular role. Ribosomal proteins are among the classes of genes that have retained the highest number of duplicates. These duplicated copies also share higher sequence similarity than duplicate pairs in other functional classes. Although this could be due to frequent gene conversion (Kellis et al. 2004), it could also be attributed to the fact that sequence variations causing changes in protein folding or surface properties would compromise assembly of the ribosome. As described below, the results of recent large-scale screens suggest that the latter model may be true.

It has been thought that duplicate ribosomal proteincoding genes are redundant in function (Rotenberg et al. 1988). The existence of duplicated ribosomal protein genes was first discovered when initial clones of ribosomal proteins were isolated (Woolford et al. 1979). Although some studies suggested that duplication was restricted to the most abundant ribosomal proteins (Fried et al. 1981), later reports indicated that the transcripts for all ribosomal proteins are expressed at similar levels, and the contributions of both copies of a duplicated ribosomal protein roughly equal the expression of ribosomal proteins encoded by a single gene (Kim and Warner 1983). As more ribosomal proteins were cloned and identified, it was observed that they typically have highly similar coding sequences but very different untranslated regions (UTRs) and introns. In general, deletion of each copy of a pair led to different effects on fitness, and the two copies were often expressed at different levels (Abovich and Rosbash 1984; Leer et al. 1984, 1985; Herruer et al. 1987; Lucioli et al. 1988; Rotenberg et al. 1988; Arevalo and Warner 1990; Yon et al. 1991; Synetos et al. 1992). The demonstration that the growth defect caused by the deletion of one copy of a given ribosomal protein gene could be rescued by the introduction of the other on a multicopy plasmid suggested that the two copies of a duplicated ribosomal protein gene were functionally redundant (Rotenberg et al. 1988).

However, a few directed studies suggest that the fitness levels being measured in previous research may not fully reflect the cellular roles of a given duplicated ribosomal protein gene. For instance, Warner (1989) noted that the fitness defect induced by the absence of a copy of certain duplicated ribosomal protein genes is not necessarily proportional to that copy's effect on expression. Furthermore, although the growth defect of rps27bs cells can be rescued by introduction of RPS27A on a multicopy plasmid, rps27as cells exhibit ribosomal assembly defects despite wild-type doubling times (Baudin-Baillieu et al. 1997). Together, these results suggest that the earlier studies of expression levels and fitness may have missed more subtle functional differences between the two copies.

High-throughput phenotypic screens have demonstrated that certain cellular processes require specific copies of duplicated ribosomal protein genes. $\mathrm{Ni}$ and Snyder (2001) noted that bud site selection requires only one paralog each of certain duplicated ribosomal protein genes. A screen for vacuolar protein sorting defects (Bonangelino et al. 2002) revealed paralog-specific requirements for ribosomal protein genes, suggesting that the two copies of certain ribosomal protein genes play dif- 
ferent roles regarding subcellular organization. Screens for genes required for centromeric cohesion, cell cycle progression, and telomeric length each yielded different subsets of duplicated ribosomal protein genes, and typically required only one of the two paralogs (Zettel et al. 2003; Askree et al. 2004; Marston et al. 2004), suggesting that one copy of certain pairs may have evolved new DNA-related functions. Similarly, a screen for genes required in sporulation and meiosis (Enyenihi and Saunders 2003) indicated that the two coies of many ribosomal proteins have different roles in these processes.

Furthermore, although not specifically discussed in previous reports, closer examination of recent large-scale phenotype screens reveals additional functional differences between the two copies of most duplicated ribosomal protein genes (S. Komili, N.G. Farny, F.P. Roth, and P.A. Silver, in prep.). In some cases, such as in treatment of cells with Wortmannin, the two copies of a given duplicated ribosomal protein gene yield opposite phenotypes: Deletion of one copy makes cells "resistant" to it (i.e., faster growing), whereas deletion of the other copy makes cells "sensitive" to it (i.e., impaired growth) (Zewail et al. 2003). Similar effects were obtained for rapamycin (Chan et al. 2000) and K1 killer toxin (Page et al. 2003). High-throughput chemical and phenotypic screens indicate that, in general, the two copies of a given duplicated ribosomal protein are required for different pathways (Begley et al. 2002; Giaever et al. 2004; Dudley et al. 2005; Parsons et al. 2006). Furthermore, unlike early studies that suggested that the ratio between two copies of a given ribosomal protein gene remain constant despite changes in extracellular conditions (Herruer et al. 1987), oligo arrays revealed that the expression levels of many duplicated ribosomal protein genes change independently in response to MMS (Jelinsky and Samson 1999; S. Komili, N.G. Farny, F.P. Roth, and P.A. Silver, in prep.).

For each of the cellular processes discussed above, the paralog required for that process is not necessarily the paralog with the most severe null mutant phenotype in rich media (Winzeler et al. 1999; S. Komili, N.G. Farny, F.P. Roth, and P.A. Silver, in prep.). Thus, fitness in rich media does not capture specialized functions of duplicated ribosomal protein genes. Moreover, the required paralog is not necessarily the most highly expressed paralog, arguing that the differential phenotypes are not simply due to gene dosage effects.

The current study by Haarer et al. (2007) has added to the evidence of subfunctionalization between the two copies of duplicated ribosomal protein genes. Twelve of the 13 ribosomal protein genes that were $\mathrm{CHI}$ with actin are duplicated. Eight of these 12 are members of a pair for which the other copy was not CHI with actin. Rhodamine-phalloidin staining confirmed that, for these pairs of duplicated ribosomal proteins, deletion of the copy that is CHI with actin leads to defects in actin and cell morphology, whereas the other copy is dispensable for actin cytoskeleton formation. As with previous studies, the copy required for actin cytoskeleton formation is not necessarily the copy expressed at a higher level, and al- though six of these ribosomal protein genes exhibit haploinsufficiency independent of actin (Deutschbauer et al. 2005), the remaining seven ribosomal proteins are only haploinsufficient in conjunction with lowered actin expression.

Taken together, there is a growing body of evidence for the subfunctionalization of duplicated ribosomal protein genes. Although some ribosomal proteins have been shown to have extraribosomal roles that include transcription, DNA repair, translational regulation, and regulation of development (Wool 1996), the fact that most of the large-scale screens found not one but many ribosomal proteins suggests that the situation is more complex for these processes and others. The recent discovery that many (but not all) ribosomal proteins act as tumor suppressors in zebrafish (Amsterdam et al. 2004) suggests that this subfunctionalization may be conserved in other organisms, and indicates the important role these ribosomal proteins may play. Unfortunately, none of these processes have a known specific requirement for translational regulation, making mechanistic insights difficult. The connection between translation and specialized roles for duplicated ribosomal genes, supported by diverse genetic evidence from Haarer et al. (2007) and others, remains a mystery.

\section{Future directions}

Further developments in large-scale reverse-genetic screening may come through advances in experimental methodology, analysis of the resulting data, or in extending these ideas to other species. For example, CHI screening might achieve greater efficiency and sensitivity by combining the approaches of Baetz et al. (2004) and Haarer et al. (2007) with dSLAM to measure fitness effects using a pool of doubly heterozygous strains under competitive growth conditions. This would eliminate the need for separate growth assays of each strain. Additionally, data analysis would be improved by a clearer definition of genetic interaction; i.e., which double-mutant phenotypes should be considered "surprising": Some large-scale studies have defined synthetic interaction by a double-mutant fitness that is below the least-fit single mutant (Tong et al. 2001, 2004; Ooi et al. 2003; Pan et al. 2006), while others define interaction by deviation of the double-mutant fitness from the product of the two single-mutant fitness measurements (Puniyani et al. 2004; R.P. St. Onge, R. Mani, J. Oh, M. Proctor, E. Fung, R. Davis, C. Nislow, F.P. Roth, and G. Giaver, unpubl.). It will also be valuable to search for UNC and $\mathrm{CHI}$ interaction in other species. Indeed, RNAi has become the modern reverse-genetic method of choice in several organisms (Sen and Blau 2006). The availability of RNAi reagents corresponding to every gene allows reverse-genetic screens to be performed in more complex organisms (e.g., see Friedman and Perrimon 2006; Lehner et al. 2006; Moffat et al. 2006), with some recent progress in double-RNAi approaches (Tischler et al. 2006). To the extent that RNAi is a "knockdown" of gene expression rather than a complete knockout, this technique may 
recover interactions due to the same gene dosage effects that lead to UNC interaction.

In summary, the current report by Haarer et al. (2007) is an exciting example of recent advances in reverse-genetic screens, and it is clear that many important developments will soon follow.

\section{Acknowledgments}

We thank P. Silver, J. Salas-Marco, R. Mani, Y. Suzuki, G. Giaver, R.P. St. Onge, C. Boone, and B. Andrews for helpful discussions. We also thank D. Muzzey for critical reading of the manuscript. This work was supported by the Harvard Biophysics Graduate Program and by NIH/NHGRI grant HG003224.

\section{References}

Abovich, N. and Rosbash, M. 1984. Two genes for ribosomal protein 51 of Saccharomyces cerevisiae complement and contribute to the ribosomes. Mol. Cell. Biol. 4: 1871-1879.

Amsterdam, A., Sadler, K.C., Lai, K., Farrington, S., Bronson, R.T., Lees, J.A., and Hopkins, N. 2004. Many ribosomal protein genes are cancer genes in zebrafish. PLOS Biol. 2: E139.

Arevalo, S.G. and Warner, J.R. 1990. Ribosomal protein L4 of Saccharomyces cerevisiae: The gene and its protein. Nucleic Acids Res. 18: 1447-1449.

Askree, S.H., Yehuda, T., Smolikov, S., Gurevich, R., Hawk, J., Coker, C., Krauskopf, A., Kupiec, M., and McEachern, M.J. 2004. A genome-wide screen for Saccharomyces cerevisiae deletion mutants that affect telomere length. Proc. Natl. Acad. Sci. 101: 8658-8663.

Baetz, K.K., Krogan, N.J., Emili, A., Greenblatt, J., and Hieter, P. 2004. The ctf13-30/CTF13 genomic haploinsufficiency modifier screen identifies the yeast chromatin remodeling complex RSC, which is required for the establishment of sister chromatid cohesion. Mol. Cell. Biol. 24: 1232-1244.

Baudin-Baillieu, A., Tollervey, D., Cullin, C., and Lacroute, F. 1997. Functional analysis of Rrp7p, an essential yeast protein involved in pre-rRNA processing and ribosome assembly. Mol. Cell. Biol. 17: 5023-5032.

Begley, T.J., Rosenbach, A.S., Ideker, T., and Samson, L.D. 2002. Damage recovery pathways in Saccharomyces cerevisiae revealed by genomic phenotyping and interactome mapping. Mol. Cancer Res. 1: 103-112.

Bonangelino, C.J., Chavez, E.M., and Bonifacino, J.S. 2002. Genomic screen for vacuolar protein sorting genes in Saccharomyces cerevisiae. Mol. Biol. Cell 13: 2486-2501.

Chan, T., Carvalho, J., Riles, L., and Zheng, X. 2000. A chemical genomics approach toward understanding the global functions of the target of rapamycin protein (TOR). Proc. Natl. Acad. Sci. 97: 13227-13232.

Collins, S.R., Schuldiner, M., Krogan, N.J., and Weissman, J.S. 2006. A strategy for extracting and analyzing large-scale quantitative epistatic interaction data. Genome Biol. 7: R63.

Davierwala, A.P., Haynes, J., Li, Z., Brost, R.L., Robinson, M.D., Yu, L., Mnaimneh, S., Ding, H., Zhu, H., Chen, Y., et al. 2005. The synthetic genetic interaction spectrum of essential genes. Nat. Genet. 37: 1147-1152.

Deutschbauer, A.M., Jaramillo, D.F., Proctor, M., Kumm, J., Hillenmeyer, M.E., Davis, R.W., Nislow, C., and Giaever, G. 2005. Mechanisms of haploinsufficiency revealed by genome-wide profiling in yeast. Genetics 169: 1915-1925.

Dudley, A.M., Janse, D.M., Tanay, A., Shamir, R., and Church, G.M. 2005. A global view of pleiotropy and phenotypically derived gene function in yeast. Mol. Syst. Biol. 1: 20050001.

Enyenihi, A.H. and Saunders, W.S. 2003. Large-scale functional genomic analysis of sporulation and meiosis in Saccharomyces cerevisiae. Genetics 163: 47-54.

Fried, H.M., Pearson, N.J., Kim, C.H., and Warner, J.R. 1981. The genes for fifteen ribosomal proteins of Saccharomyces cerevisiae. J. Biol. Chem. 256: 10176-10183.

Friedman, A. and Perrimon, N. 2006. A functional RNAi screen for regulators of receptor tyrosine kinase and ERK signalling. Nature 444: 230-234.

Fuller, M.T., Regan, C.L., Green, L.L., Robertson, B., Deuring, R., and Hays, T.S. 1989. Interacting genes identify interacting proteins involved in microtubule function in Drosophila. Cell Motil. Cytoskeleton 14: 128-135.

Giaever, G., Chu, A.M., Ni, L., Connelly, C., Riles, L., Veronneau, S., Dow, S., Lucau-Danila, A., Anderson, K., Andre, B., et al. 2002. Functional profiling of the Saccharomyces cerevisiae genome. Nature 418: 387-391.

Giaever, G., Flaherty, P., Kumm, J., Proctor, M., Nislow, C., Jaramillo, D.F., Chu, A.M., Jordan, M.I., Arkin, A.P., and Davis, R.W. 2004. Chemogenomic profiling: Identifying the functional interactions of small molecules in yeast. Proc. Natl. Acad. Sci. 101: 793-798.

Gresham, D., Ruderfer, D.M., Pratt, S.C., Schacherer, J., Dunham, M.J., Botstein, D., and Kruglyak, L. 2006. Genomewide detection of polymorphisms at nucleotide resolution with a single DNA microarray. Science 311: 1932-1936.

Haarer, B., Viggiano, S.,Hibbs, M.A., Troyanskaya, O.G., and Amberg, D.C. 2007. Modeling complex genetic interactions in a simple eukaryotic genome: Actin displays a rich spectrum of complex haploinsufficiencies. Genes \& Dev. (this issue).

Hawley, R. and Walker, M. 2003. Advanced genetic analysis: Finding meaning in a genome. Oxford, Blackwell, Malden, MA.

Hays, T.S., Deuring, R., Robertson, B., Prout, M., and Fuller, M.T. 1989. Interacting proteins identified by genetic interactions: A missense mutation in $\alpha$-tubulin fails to complement alleles of the testis-specific $\beta$-tubulin gene of Drosophila melanogaster. Mol. Cell. Biol. 9: 875-884.

Herruer, M.H., Mager, W.H., Woudt, L.P., Nieuwint, R.T., Wassenaar, G.M., Groeneveld, P., and Planta, R.J. 1987. Transcriptional control of yeast ribosomal protein synthesis during carbon-source upshift. Nucleic Acids Res. 15: 1013310144.

Hieronymus, H., Yu, M.C., and Silver, P.A. 2004. Genome-wide mRNA surveillance is coupled to mRNA export. Genes \& Dev. 18: 2652-2662.

Jelinsky, S. and Samson, L. 1999. Global response of Saccharomyces cerevisiae to an alkylating agent. Proc. Natl. Acad. Sci. 96: 1486-1491.

Kellis, M., Birren, B.W., and Lander, E.S. 2004. Proof and evolutionary analysis of ancient genome duplication in the yeast Saccharomyces cerevisiae. Nature 428: 617-624.

Kim, C.H. and Warner, J.R. 1983. Messenger RNA for ribosomal proteins in yeast. J. Mol. Biol. 165: 79-89.

Le Goff, X., Philippe, M., and Jean-jean, O. 1997. Overexpression of human release factor 1 alone has an antisuppressor effect in human cells. Mol. Cell. Biol. 17: 3164-3172.

Leer, R.J., van Raamsdonk-Duin, M.M., Mager, W.H., and Planta, R.J. 1984. The primary structure of the gene encoding yeast ribosomal protein L16. FEBS Lett. 175: 371-376.

Leer, R.J., van Raamsdonk-Duin, M.M., Molenaar, C.M., Witsenboer, H.M., Mager, W.H., and Planta, R.J. 1985. Yeast contains two functional genes coding for ribosomal protein S10. Nucleic Acids Res. 13: 5027-5039. 
Lehner, B., Crombie, C., Tischler, J., Fortunato, A., and Fraser, A.G. 2006. Systematic mapping of genetic interactions in Caenorhabditis elegans identifies common modifiers of diverse signaling pathways. Nat. Genet. 38: 896-903.

Lucioli, A., Presutti, C., Ciafre, S., Caffarelli, E., Fragapane, P., and Bozzoni, I. 1988. Gene dosage alteration of L2 ribosomal protein genes in Saccharomyces cerevisiae: Effects on ribosome synthesis. Mol. Cell. Biol. 8: 4792-4798.

Marston, A.L., Tham, W.H., Shah, H., and Amon, A. 2004. A genome-wide screen identifies genes required for centromeric cohesion. Science 303: 1367-1370.

Milgrom, E., West Jr., R.W., Gao, C., and Shen, W.C. 2005. TFIID and Spt-Ada-Gen5-acetyltransferase functions probed by genome-wide synthetic genetic array analysis using a Saccharomyces cerevisiae taf9-ts allele. Genetics 171: 959-973.

Moffat, J., Grueneberg, D.A., Yang, X., Kim, S.Y., Kloepfer, A.M., Hinkle, G., Piqani, B., Eisenhaure, T.M., Luo, B., Grenier, J.K., et al. 2006. A lentiviral RNAi library for human and mouse genes applied to an arrayed viral high-content screen. Cell 124: 1283-1298.

Ni, L. and Snyder, M. 2001. A genomic study of the bipolar bud site selection pattern in Saccharomyces cerevisiae. Mol. Biol. Cell 12: 2147-2170.

Ooi, S.L., Shoemaker, D.D., and Boeke, J.D. 2003. DNA helicase gene interaction network defined using synthetic lethality analyzed by microarray. Nat. Genet. 35: 277-286.

Page, N., Gerard-Vincent, M., Menard, P., Beaulieu, M., Azuma, M., Dijkgraaf, G., Li, H., Marcoux, J., Nguyen, T., Dowse, T., et al. 2003. A Saccharomyces cerevisiae genome-wide mutant screen for altered sensitivity to K1 killer toxin. Genetics 163: $875-894$

Pan, X., Ye, P., Yuan, D.S., Wang, X., Bader, J.S., and Boeke, J.D. 2006. A DNA integrity network in the yeast Saccharomyces cerevisiae. Cell 124: 1069-1081.

Parsons, A.B., Lopez, A., Givoni, I.E., Williams, D.E., Gray, C.A., Porter, J., Chua, G., Sopko, R., Brost, R.L., Ho, C.H., et al. 2006. Exploring the mode-of-action of bioactive compounds by chemical-genetic profiling in yeast. Cell 126: 611625.

Presutti, C., Ciafre, S.A., and Bozzoni, I. 1991. The ribosomal protein L2 in S. cerevisiae controls the level of accumulation of its own mRNA. EMBO J. 10: 2215-2221.

Puniyani, A., Liberman, U., and Feldman, M.W. 2004. On the meaning of non-epistatic selection. Theor. Popul. Biol. 66: $317-321$.

Rotenberg, M.O., Moritz, M., and Woolford Jr., J.L. 1988. Depletion of Saccharomyces cerevisiae ribosomal protein L16 causes a decrease in $60 \mathrm{~S}$ ribosomal subunits and formation of half-mer polyribosomes. Genes \& Dev. 2: 160-172.

Salas-Marco, J. and Bedwell, D. 2004. GTP hydrolysis by eRF3 facilitates stop codon decoding during eukaryotic translation termination. Mol. Cell. Biol. 24: 7769-7778.

Schuldiner, M., Collins, S.R., Thompson, N.J., Denic, V., Bhamidipati, A., Punna, T., Ihmels, J., Andrews, B., Boone, C., Greenblatt, J.F., et al. 2005. Exploration of the function and organization of the yeast early secretory pathway through an epistatic miniarray profile. Cell 123: 507-519.

Sen, G.L. and Blau, H.M. 2006. A brief history of RNAi: The silence of the genes. FASEB J. 20: 1293-1299.

Sopko, R., Huang, D., Preston, N., Chua, G., Papp, B., Kafadar, K., Snyder, M., Oliver, S.G., Cyert, M., Hughes, T.R., et al. 2006. Mapping pathways and phenotypes by systematic gene overexpression. Mol. Cell 21: 319-330.

Stark, C., Breitkreutz, B.J., Reguly, T., Boucher, L., Breitkreutz, A., and Tyers, M. 2006. BioGRID: A general repository for interaction datasets. Nucleic Acids Res. 34: D535-D539.

Stearns, T. and Botstein, D. 1988. Unlinked noncomplementation: Isolation of new conditional-lethal mutations in each of the tubulin genes of Saccharomyces cerevisiae. Genetics 119: 249-260.

Stevenson, L.F., Kennedy, B.K., and Harlow, E. 2001. A largescale overexpression screen in Saccharomyces cerevisiae identifies previously uncharacterized cell cycle genes. Proc. Natl. Acad. Sci. 98: 3946-3951.

Synetos, D., Dabeva, M.D., and Warner, J.R. 1992. The yeast ribosomal protein S7 and its genes. J. Biol. Chem. 267: 30083013.

Tischler, J., Lehner, B., Chen, N., and Fraser, A.G. 2006. Combinatorial RNA interference in C. elegans reveals that redundancy between gene duplicates can be maintained for more than 80 million years of evolution. Genome Biol. 7: R69.

Tong, A.H., Evangelista, M., Parsons, A.B., Xu, H., Bader, G.D. Page, N., Robinson, M., Raghibizadeh, S., Hogue, C.W., Bussey, H., et al. 2001. Systematic genetic analysis with ordered arrays of yeast deletion mutants. Science 294: 2364-2368.

Tong, A.H., Lesage, G., Bader, G.D., Ding, H., Xu, H., Xin, X., Young, J., Berriz, G.F., Brost, R.L., Chang, M., et al. 2004. Global mapping of the yeast genetic interaction network. Science 303: 808-813.

Vilardell, J. and Warner, J.R. 1997. Ribosomal protein L32 of Saccharomyces cerevisiae influences both the splicing of its own transcript and the processing of rRNA. Mol. Cell. Biol. 17: 1959-1965.

Warner, J.R. 1989. Synthesis of ribosomes in Saccharomyces cerevisiae. Microbiol. Rev. 53: 256-271.

Winzeler, E.A., Shoemaker, D.D., Astromoff, A., Liang, H., Anderson, K., Andre, B., Bangham, R., Benito, R., Boeke, J.D., Bussey, H., et al. 1999. Functional characterization of the $S$. cerevisiae genome by gene deletion and parallel analysis. Science 285: 901-906.

Wool, I. 1996. Extraribosomal functions of ribosomal proteins. Trends Biochem. Sci. 21: 164-165.

Woolford Jr., J.L., Hereford, L.M., and Rosbash, M. 1979. Isolation of cloned DNA sequences containing ribosomal protein genes from Saccharomyces cerevisiae. Cell 18: 1247-1259.

Yon, J., Giallongo, A., and Fried, M. 1991. The organization and expression of the Saccharomyces cerevisiae L4 ribosomal protein genes and their identification as the homologues of the mammalian ribosomal protein gene L7a. Mol. Gen. Genet. 227: 72-80.

Yook, K.J., Proulx, S.R., and Jorgensen, E.M. 2001. Rules of nonallelic noncomplementation at the synapse in Caenorhabditis elegans. Genetics 158: 209-220.

Zettel, M.F., Garza, L.R., Cass, A.M., Myhre, R.A., Haizlip L.A., Osadebe, S.N., Sudimack, D.W., Pathak, R., Stone, T.L., and Polymenis, M. 2003. The budding index of Saccharomyces cerevisiae deletion strains identifies genes important for cell cycle progression. FEMS Microbiol. Lett. 223: 253-258.

Zewail, A., Xie, M., Xing, Y., Lin, L., Zhang, P., Zou, W., Saxe, J., and Huang, J. 2003. Novel functions of the phosphatidylinositol metabolic pathyway discovered by a chemical genomics screen with wortmannin. Proc. Natl. Acad. Sci. 100 3345-3350. 


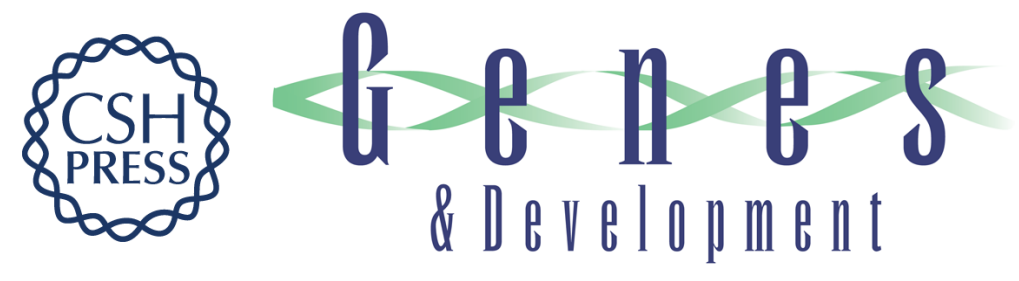

\section{Genetic interaction screens advance in reverse}

Suzanne Komili and Frederick P. Roth

Genes Dev. 2007, 21:

Access the most recent version at doi:10.1101/gad.1512707

Related Content Modeling complex genetic interactions in a simple eukaryotic genome: actin displays a rich spectrum of complex haploinsufficiencies

Brian Haarer, Susan Viggiano, Mathew A. Hibbs, et al.

Genes Dev. January, 2007 21: 148-159

References This article cites 62 articles, 33 of which can be accessed free at: http://genesdev.cshlp.org/content/21/2/137.full.html\#ref-list-1

Articles cited in:

http://genesdev.cshlp.org/content/21/2/137.full.html\#related-urls

\section{License}

Email Alerting

Service

Receive free email alerts when new articles cite this article - sign up in the box at the top right corner of the article or click here.

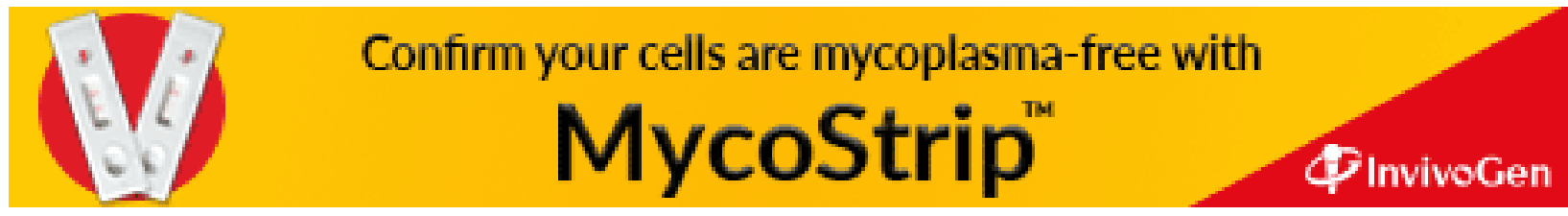

